Methods: To model the NHEJ repair mechanism we used the host-cell reactivation assay. A luciferase reporter vector pGL3-luc was damaged with endonuclease and etoposide, and then introduced into E1A+Ras-transformed and normal (NIH3T3) mouse fibroblasts, using lipofectamine transfection. Transfected cells were incubated in the presence or absence of HDACi sodium butyrate and afterwards, the efficiency of DNA repair was evaluated by measurement of the luciferase activity. Results: Double-strand breaks, which were introduced by a damaging agent or an endonuclease into the plasmid DNA, decreased luciferase transcription. However, it reclaimed afterwards owing to DNA repair. We have shown that the recovery of DNA has occurred less efficiently in the presence of sodium butyrate in transformed cells, while in normal fibroblasts sodium butyrate did not affect the NHEJ-repair efficiency. Further, the HDACi suppressed the expression of repair proteins $(\mathrm{Ku} 80, \mathrm{Ku} 70, \mathrm{Mre} 11)$ in transformed, but not in normal cells. Conclusions: We have thus demonstrated a fundamental difference between the influence of HDACi on DNA repair via NHEJmechanism in oncogene-transformed and in normal cells. DNA repair capability of E1A+Rastransformed cells was reduced by HDACi, but in normal cells it stays unaffected. This effect is very useful for anticancer chemotherapy since HDACi could be applied in combination with DNA damage agents.

Acknowledgments: A project is financed by RSF 19-75-10060.

References: 1. Wang Y. et al., Cancer Lett. 2017; 400: 47-60; 2. Sun G. et al., Journal of cancer 2015; 6(10): 996-1004; 3. Rossiello F. et al., Curr Opin Genet Dev. 2014;26: 89-95; 4. Munshi, A. et al. Clin. Cancer Res. 2005; 11: 4912-22; 5. Abramova, M. V. et al. Cancer Biol Ther. 2011; 12(12): 1069-77. doi: http://dx.doi.org/10.7124/bc.0009E1

\section{G-3. Nuclear protein phosphatase Wip1 regulate sensitivity of human colorectal cancer cells to DNA damaging anti-cancer agents}

Bogdan B. Grigorash $^{1,2}$, B. Uyanik ${ }^{1}$, Elena Yu. Kochetkova ${ }^{2}$, Oleg N. Demidov' ${ }^{1,2}$

${ }^{1}$ INSERM 1231, University of Burgundy FrancheComte, Dijon, France; ${ }^{2}$ Institute of Cytology RAS, Saint-Petersburg, Russia

bogdgrig@gmail.com

To evaluate impact of nuclear wild-type p53 induced phosphatase Wip1 on sensitivity of human colorectal cancer cell line DLD1 to the DNA damaging drugs: 5-fluouracil and oxaliplatin. Methods: Human colorectal cell line DLD1 with wild-type p53 status was used for all experiments. Stable cell line with overexpression of Wip1 (DLD1-Wip1ON) was derived using lentiviral transduction of DLD1 cells with viral particles containing human Wip 1 cDNA under control of EF1a promoter. Wip1 overexpression was validated by immunoblotting. Wip1 gene knockout was performed using CRISPR/Cas9 approach. Briefly, plasmids encoding three gRNAs targeting $2 \mathrm{nd}$, 3 rd and 5th exon of Wip1 gene were co-transfected with three donor plasmids to produce the knock-in of selection cassette via homologous recombination. Proper cassette integration and disruption of Wip1 coding sequence were validated by PCR. Cellular toxicity was estimated by MTT essay, senescence - by SAb-gal staining, cycle - by 7-AAD staining. Results: We showed that overexpression of Wip1 led to phosphatase accumulation in the nucleus and affected DNA damage response 
in colorectal cancer cells by decreasing the sensitivity of DLD1 cells to combination anticancer drugs used in clinics for the treatment of colorectal cancer, oxaliplatin and 5-fluouracil. Analysis of accumulation of senescenceassociated beta-galactosidase showed that neither DLD1, nor DLD1-Wip1ON cells don not develop senescent phenotype and their resistance is not based on induction of senescence. To prove that resistance of DLD1-Wip1ON cells occur due to Wip1 overexpression, we created DLD1-Wip1KO cell line with bi-allellic knockout of Wip1 gene. The opposite effect was observed in cells with deletion of Wip1 making them more sensitive to combination of DNA damaging drugs. Conclusions: Overexpression and deletion of nuclear phosphatase Wip1 affects DNA damage response in colorectal cancer cells induced by a classical combination of chemotherapeutic drugs used for FOLFOX adjuvant therapy of colorectal cancer.

The reported study was funded by RFBR according to the research project № 17-04-01592.

doi: http://dx.doi.org/10.7124/bc.0009E2

\section{G-4. A novel approach in studies of the posttranslational modification effect on YB-1 translocation}

\section{E. M. Grigorieva, D. A. Mordovkina,}

E. R. Kim, L. P. Ovchinnikov

Institute of Protein Research, Russian Academy of Sciences, Pushchino, Russia

kategrigoreva@vega.protres.ru
The Y-box binding protein 1 (YB-1) is a multifunctional protein. In the cytoplasm, it is involved in mRNA translation and stabilization, while in the nucleus it participates in replication regulation and DNA transcription. Mostly, YB-1 shows the cytoplasmic localization, but in some cases (under stress or modification) it goes to the nucleus. Its best studied nuclear translocation-causing modification is phosphorylation at S102. The in vitro transport assay technique is a simple and convenient method to study the mechanisms of protein transport. Its advantage is the possibility to control transport conditions. Phosphorylation is a posttranslational modification that regulates many cellular events including protein translocation. To study its effect, a phosphomimetic technique is used, in which amino acid substitutions are introduced into phosphorylation sites to simulate the dephosphorylated state (replacement by Ala) and the phosphorylated state (by Asp or Glu). We used a combination of these two techniques to find out the effect of YB-1 modifications on its nucleus-cytoplasm transport. Methods The sitedirected mutagenesis was used to make mutations in the YB-1 phosphorylation sites. The proteins were isolated and the transport assay system was assembled according to [1,2]. For the import reaction, we used YB-1 with S102 replaced by alanine or aspartic acid. Protein localization was assessed by immunofluorescence microscopy. Results In the transport assay system, two types of cell culture conditions were used: standard conditions (YB-1 in the cytoplasm) and transition-stimulating conditions (YB-1 in the nucleus). If phosphorylation stimulates the transition, the phosphomimetic mutant will show the nuclear localization even in standard conditions, while the dephosphory- 